\title{
Balances y aproximaciones teóricas al movimiento estudiantil
}

\section{Balances and theoretical approaches to the student}

movement

\author{
Gerónimo Ontiveros Juárez* \\ Francisco Alberto Pérez Piñón ${ }^{* * *}$
}

\begin{abstract}
* Profesor de telebachillerato en las Varas Municipio de Madera, Chihuahua (México). Es Licenciado en Filosofia por la Facultad de Filosofia y Letras de la Universidad Autónoma de Chihuahua; Maestría en Educación por la Universidad Pedagógica Nacional de Ciudad Madera; estudiante becario del Doctorado en Educación, Artes y Humanidades de la Facultad de Filosofía y Letras de la Universidad Autónoma de Chihuahua. Realiza actividades académicas y de investigación en el programa acreditado por Consejo Nacional de Ciencia y Tecnología. Correo electrónicogeroontiveros@gmail.com
\end{abstract}

(D) https://orcid.org/0000-0003-4314-8358

**Profesor investigador de la Universidad Autónoma de Chihuahua (México). Es doctor en Ciencias Pedagógicas (Cuba). Entre sus publicaciones recientes están: "Apuntes para una historia posmodernista" en Espacio abierto vol. 25, n.4 (2016); capitulo de libro "La hacienda de coyotillos, un acercamiento a los contenidos educativos en Acercamientos a la historia de la educación. Diálogos, actores y fuentes en la construcción del conocimiento histórico (2017). Trabaja la línea de investigación de Historia e Historiografía de la Educación. Cuenta con reconocimientos al perfil Prodep y del Sistema Nacional de Investigadores Nivel 1. Es miembro de la Sociedad Mexicana de Historia de la Educación, del Consejo Mexicano de Investigación Educativa y de la Red de Investigadores Educativos Chihuahua. Correo electrónico aperezp@uach.mx

https://orcid.org/0000-0003-4316-6484

Historial editorial

Recibido: 16-mayo-2018

Primera revisión: 22-mayo-2018

Segunda revisión: 30-agosto-2018

Aceptado: 10-septiembre-2018

Publicado: 31-octubre-2018 


\section{Balances y aproximaciones teóricas al movimiento estudiantil}

\section{Resumen}

Los movimientos estudiantiles se siguen produciendo en momentos indeterminados, las causas y mecanismos que los ocasionan parecen ser desconocidos a primera vista. Las explicaciones dadas los atribuyen principalmente a factores ideológicos y políticos, lo que va en detrimento de la importancia de este fenómeno social. Se trata entonces de encuadrarlos y discriminar las categorías de análisis a que pertenecen sus datos. Este trabajo se desprende de una investigación de tesis doctoral, el objetivo es, mediante la narrativa, conseguir una aproximación en la que el lector distinga su forma epistemológica. La pregunta por responder es ¿Por qué se origina una manifestación estudiantil? La respuesta se dará por medio de un análisis crítico de interpretación de las fuentes y de los trabajos de especialistas. Los resultados han mostrado que los movimientos estudiantiles han acompañado la vida universitaria desde su creación, pero siguen siendo marginados en la historia oficial. El investigador científico de los movimientos estudiantiles debe comprender este proceso, no dando su trabajo por terminado, sino parcialmente y nunca de modo determinante. Y desde las posibles interpretaciones de la historia, abrir una base filológicamente sustentable para el estudio de su complejidad, mediante otras ramas del conocimiento.

40 Palabras Clave: Análisis histórico, autoridades universitarias, movimientos estudiantiles, reforma universitaria. 


\title{
Balances and theoretical approaches to the student movement
}

\begin{abstract}
Student movements occur at indefinite moments and their causes seem to be unknown. They are commonly given political and ideological explanations, being this a detriment to the importance of this social phenomenon. Hence, they need to be framed and their data categories need to be distinguished. This paper emerges from a doctoral thesis and seeks to obtain, through narrative, an approach for the reader to identify the epistemology behind student movements. It attempts to answer the research question why do student demonstrations occur? through a critical analysis of the interpretation of the sources and research of specialists in the area. Findings suggest that student movements have existed since the creation of university, but that they are still marginalized in official history. It is necessary that researchers of student movements understand this process, by not thinking of their research as definite but rather as partially completed, and that they find a philologically sustainable basis from the possible interpretations of history in the study of their complexity through other fields of knowledge.
\end{abstract}

Keywords: Historical analysis, university authorities, student movements, university reform. 


\section{Introducción}

Después de un acontecimiento dramático es común que los protagonistas que viven las convulsiones de la época narren o escriban al respecto. Esto ocurrió al finalizar el siglo XVIII, cuando muchos de los llamados Ilustrados comenzaron la tarea de reconstruir su pasado inmediato. Poco después de la Revolución Francesa, no faltaron intentos para explicar posibles causas de los sucesos que culminaron con la Toma de la Bastilla, comenzando por la escasez de semillas hasta la muerte de Marat; vistos a manera de "efecto dominó" que se produjo primero en Francia y se extendió al resto de Europa.

La entrada a la Época Contemporánea no fue del todo un ingreso festivo, más que eso, fue una era en que el caos ilustrado azotaba el país de la liberté. En el artículo “¿Cómo ha sido la vuelta?”, Diego Sánchez realiza un bosquejo general del papel protagónico que tomaron las universidades francesas a partir del frenesí libertario:

Pasada la Revolución Francesa, París era un febril hervidero de nuevas ideas. Jacobinos, volterianos, girondinos, hebertistas, enragés, rousseaunianos, radicales, enciclopedistas, racionalistas, masones, republicanos, agitadores, idealistas, subversivos, rebeldes y librepensadores, se mezclan en un asombroso caos de democracia montonera, de chusma ilustrada e insurrecta, y universal, como base de cualquier cambio duradero. Fue ese ímpetu de volcán incendiario el que permitió que el 23 de octubre de I794 se estableciera la Ecole del 'de Marte por el Comité de Salud Pública y a partir de aquí se multiplicaran los liceos y escuelas públicas; y con ellos, las protestas estudiantiles. En I829, en París, los estudiantes paran las clases urgiendo una educación laica [González, 2010, p. II5]. 
Aunque las universidades en Francia no tuvieron un papel central en la caída del antiguo régimen, las nuevas ideas de libertad, derechos humanos, democracia, derecho a la educación laica y pública, a la justicia social, así como la fraternidad, fueron ideas que comenzaron a tener un impacto trascendental en toda Francia y en el viejo continente. La modernización llegó a las cúpulas del poder burgués y se modernizó la educación superior. Esto tuvo como consecuencia la organización estudiantil más importante en la historia contemporánea del mundo occidental.

Al finalizar el siglo XVIII, las ideas de la Ilustración habían permeado completamente en la población francesa, al grado de comenzar a hacer estragos irreversibles en las estructuras sociales, no sólo de Francia y del continente europeo, sino del mundo entero (suceso que concluyó con la destrucción de la monarquía en toda la línea). Paradójicamente, las órdenes eclesiásticas en Latinoamérica continuaron impulsando la formación de profesionistas universitarios con el objetivo de mantener su hegemonía política y así combatir las ideas de la Ilustración. La incorporación de la ciencia en las universidades coloniales fue posiblemente una contienda que sostenían órdenes religiosas hostiles a la hegemonía de la iglesia católica (concretamente, la llamada Compañía de Jesús).

\section{Raíces de las universidades latinoamericanas}

En 1538 se inaugura la primera universidad latinoamericana en Santo Domingo, en I55I la de Lima en Perú y en 5533 la de la Ciudad de México. Estas dos últimas instituciones recibieron pocos años después el título de "Real y Pontificia", respondiendo a la conformación de los estados pre-modernos, donde los gobiernos virreinales apostaban la grandeza 
de sus naciones a la construcción y consolidación de una educación de orden superior. Este proyecto apuntaba a la formación de profesionistas ilustrados, pero fieles a la monarquía para que dieran forma y solidez a los nuevos Estados coloniales.

Parece que el conocimiento, por su propia naturaleza, va generando en el individuo un deseo de saber cada vez más en profundidad, de tal manera que se cuestiona sobre sí mismo. La integración de planes y programas en las universidades europeas -donde las nuevas teorías científicas habían alcanzado un grado de criticidad- generaron un movimiento intelectual en torno a estos nuevos postulados, introduciéndose en las universidades de reciente creación de América Latina.

Así, se puede decir que el nivel de la enseñanza universitaria en el siglo XVIII no iba muy a la zaga del nivel universitario en Europa. Como muestra de lo que decimos, pueden citarse los resultados de una investigación sobre la Universidad de San Carlos en Guatemala; según estos resultados, la revisión de las tesis presentadas en esta Universidad permiten concluir que desde el Discurso del Método de un Descartes o la Ley de Gravitación de Isaac Newton hasta los ensayos de Franklin sobre electricidad y los últimos progresos en hidráulica, no hubo casi ningún problema que no hubiera sido defendido o investigado críticamente en cualquier examen de la Universidad de San Carlos en Guatemala [Lanning, 1956, citado por Silva y Sonntag, 44 I970, p. II5].

Las universidades de corte religioso continuaron fundándose en América Latina, siguiendo el patrón de reproducción ideológica que dictaba la corona española, pero su desarrollo histórico se diferenció profundamente del que se estaba llevando a cabo en el sistema 
universitario de Europa y Estados Unidos. Mientras que la educación latinoamericana mantenía una orientación eminentemente social, la educación norteamericana se orientaba más hacia el pragmatismo. Las diferencias entre los sistemas universitarios son una realidad, sin embargo, intentar realizar una comparación entre la educación universitaria de Estados Unidos con la latinoamericana o la europea es una labor imposible:

Las diferencias entre Paris y Bolonia todavía se reflejan en los contrastes que existen entre la organización universitaria norteamericana y la latinoamericana. La universidad medieval de París estaba controlada por los profesores, y su modelo influyó en las universidades del norte de Europa, que incluían Inglaterra y Escocia y, por tanto, indirectamente influyeron también en el modelo norteamericano. La universidad medieval de Bolonia, controlada formalmente por los estudiantes, influyó sobre las universidades del sur de Europa, que incluían Salamanca y Alcalá, las cuales fueron el modelo para las universidades españolas coloniales en el Nuevo Mundo. Los dos modelos cambiaron en el Nuevo Mundo, pero, como dice Benjamín: "los conceptos básicos de una universidad de maestros que dirigían a estudiantes, por una parte, y una universidad de estudiantes en la que los profesores eran empleados, por la otra... siguieron persistiendo [Waggoner, I973, p.2].

Los objetivos de las universidades latinoamericanas, con respecto a la norteamericana, se dan precisamente con la disyuntiva de intervención o neutralidad frente a la realidad social, lo que está directamente relacionada con el tema de la autonomía universitaria. Para George Waggoner, la reforma universitaria ocurrida en Córdoba de I9I8 asunto que se aborda más adelante- tiene indicativos de progreso más 
que de retrocesos; por el contrario, los gobiernos norteamericanos interpretan los movimientos estudiantiles latinoamericanos como la fase de una decadencia de este sistema universitario.

Las universidades latinoamericanas han creado un concepto de gobierno universitario que es independiente, estudiantil, democrático y de gran valor, pero que padece de una serie de procedimientos, procesos y estructuras académicas inefectivas y muy poco adecuadas a las responsabilidades contemporáneas de la universidad; en contraste, las universidades norteamericanas han creado procesos y procedimientos académicos efectivos y adecuados a las actuales necesidades de la enseñanza y la investigación, pero han definido mal sus procesos internos de toma de decisiones y sus relaciones con la sociedad [Waggoner, I973, p. 2].

Los ideales de libertad e independencia de los países colonizados, aunados a sus propias raíces sureñas medievales, en las cuales "Las universidades católicas de la América Latina colonial mantuvieron su autonomía -según el modelo de Salamanca y Alcalá- en el sentido que los profesores elegían sus propias autoridades" (Waggoner, I973, p. 8), influyeron grandemente en los anhelos de una autonomía universitaria que se mantuvo después de la independencia de los países latinoamericanos y de la consecuente secularización de los Estados.

\section{El alcance de la Reforma Universitaria de Córdoba}

El siglo XX despertó con las agitaciones de la Universidad de Córdoba, Argentina. Las ideas de la ilustración parecían seguir vigentes en la memoria latinoamericana. La libertad volvió a incorporarse en los discursos universitarios de la época, signados por el contexto social que 
vivían los países coloniales; por eso, las libertades democráticas se discutían en los pasillos de esta Universidad. La agitación comienza con la pugna de los estudiantes en contra de un tipo de educación monástica vertical, ya que en el centro de esta formación sólo aparecía el docente y el texto, relegando a un segundo plano el papel del estudiante como sujeto activo.

El movimiento estudiantil cordobés exigía una reforma universitaria, teniendo como fundamento de sus demandas el "Manifiesto Liminar". Esto inauguró un largo período de movimientos universitarios latinoamericanos, que comenzaron a propugnar por reformas que favorecieran la participación activa de las universidades y de los estudiantes en la solución de los problemas sociales de su propio contexto; una real y verdadera autonomía, democracia dentro y fuera de las escuelas, lo que iba en detrimento del control estatal y clerical. Estos planteamientos ponían indefectiblemente como centro de debate político a la autonomía universitaria, sellando históricamente su participación activa como agente de cambio.

La reforma de Córdoba es el precedente inmediato de una nueva modalidad en los movimientos estudiantiles, en la que el estudiantado defendía su derecho por la participación en la edificación educativa. Estas luchas se manifiestan a lo largo del siglo XX, agudizándose en los años sesenta y cerrando el siglo con la huelga de la Universidad Nacional Autónoma de México (UNAM) de 1999. El movimiento de Reforma Universitaria de Córdoba es relevante en la historia de estos conflictos. Es a partir de aquí que los movimientos estudiantiles se configuran como los nuevos protagonistas de las luchas sociales, tanto por las cualidades del estudiantado, como por la cantidad de matrícula.

Las grandes similitudes que mantienen los movimientos en universidades de distintos países, llaman la atención. Aparentemente 
no sostienen ningún tipo de conexión (comunicación) entre ellas. Incluso van a mantener algunas semejanzas a pesar de no ocurrir durante el mismo siglo. Las diferencias siguen agregándose (zona geográfica, nivel económico) pero serán las similitudes las que perdurarán a pesar de las ideologías hegemónicas que determinan su espacio, su tiempo y su cultura, las que sugieren que dichos sucesos pueden tratarse como un cuerpo sustancial, propio al estudio y no sólo adjetivamente.

Berkeley: el surgimiento de los movimientos estudiantiles de los años sesenta

Un primer acercamiento empírico a los movimientos estudiantiles nos arroja que estos han tenido una relación intrínseca con los movimientos sociales y una visión muchas veces progresiva de la sociedad. Responden a aspiraciones emanadas de propuestas democráticas y si no, por lo menos de mayores alcances de los que se está acostumbrado a reproducir sistemáticamente; estos movimientos obedecen directamente a los cambios que se realizan en una sociedad determinada, relacionados directamente con las ideas de progreso expresadas a nivel mundial. Por ejemplo, las libertades individuales que en Francia eran socializadas dentro de la comunidad estudiantil, como las ideas de paz e igualdad, fueron piezas fundamentales entre los jóvenes estudiantes en las universidades de Estados Unidos.

En 1964, la Universidad de Berkeley, California, inicia las movilizaciones estudiantiles que conmocionaron los años sesenta y setenta en el mundo. Nuevamente la libertad se estaba poniendo en riesgo y los universitarios se organizaron para defenderla; el lema fue "Free Speech Movement": una reacción estudiantil en contra de la 
imposición de un reglamento que contradecía la Constitución de los Estados Unidos de América.

Después de este primer acontecimiento en la Unión Americana surgieron en otros países movimientos similares en los que se revindicaban los derechos estudiantiles, tomándose como banderas de lucha -por ejemplo- el derecho a la autodeterminación de los pueblos, la paz, la libertad, los derechos civiles, entre otros. No sólo en el continente europeo, sino también en Asia y América, las protestas fueron multiplicándose, mientras que la respuesta de los gobiernos fue violenta y desmedida.

\section{México 68: la historia oculta}

En el dramático caso del movimiento estudiantil de 1968 en México, en el que las manifestaciones habían puesto en jaque al gobierno de Gustavo Díaz Ordaz, la respuesta del Estado a los problemas políticos fue una represión desmedida, con cientos de desaparecidos y decenas de muertos, encarcelamientos ilegales y persecuciones extrajudiciales. Es uno de los capítulos más sangrientos y terribles en la historia del México moderno. Esta represión ocurrió el 2 de octubre en Tlatelolco y no figuró en la historia oficial que le siguió a todo el periodo de los gobiernos bonapartistas ¿ ¿Por qué? Renate Marsiske apunta que "la historia de las universidades son historias institucionales, en una historia institucional no caben los conflictos, porque los conflictos según la sociedad, según las autoridades, son un estorbo en el camino común y corriente de la institución" (IISUE, 2015). Esta afirmación es una constante para quienes hablan o escriben sobre las instituciones educativas en plataformas distintas a la universitaria, normalmente otra institución que forma parte de sus allegados o soportadores oficiales 
(caso de los periódicos con mayor circulación). Ocultan implacablemente cualquier acaecimiento histórico de tipo social que se haya presentado en dichas instituciones, ocasionando así una brecha posible al conocimiento real de la propia institución.

Ya expuesta esta verticalidad oficial en el sistema universitario, que puede justificarse recordando cómo la universidad es parte del Estado, que su nacimiento es noble a la vez que conservador y posteriormente capitalista (caso de las universidades privadas o de la privatización que sufren las públicas, adecuando perfiles a los demandantes del trabajo) señalamos como una necesidad que se rescate el estudio mismo de los movimientos estudiantiles como asunto importante del país, que atañe a toda construcción histórico social, pues son fenómenos que afectan directamente la vida de las comunidades en que existen y hasta donde se expanden, por medio de su conocimiento, reconocimiento y autoridad.

Es responsabilidad que cada expedición investigadora exija explicaciones basadas en estudios académicos y de carácter científico de estos hechos y que las abastezca. Partiendo de una investigación inductivo-deductiva, que inicie con el estudio particular de un conflicto, podamos entender los mecanismos que generan los generan, posibilitando explicaciones científicas a un lector no especializado, pero interesado en el tema.

Se requiere explicar elementos que sirvan como esclarecedores a la 50 persona común, incorpora la investigación al flujo divulgador y devolviendo la conciencia al ente social. Es indispensable comprender un movimiento estudiantil, tanto en comunidades rurales o semirurales, como en una ciudad. Estas explicaciones científicas guardan distancia a las descalificaciones o a prejuicios ideológicos que puedan tergiversar de manera intencional la realidad de los fenómenos 
sociales. Por eso el investigador científico de los movimientos estudiantiles debe entenderse en proceso (cuando no a contracorriente), no dando su trabajo por terminado, sino parcialmente y nunca de modo determinante. Y desde las posibles interpretaciones de la historia, abrir una base filosóficamente sustentable para el estudio de su complejidad, por medio de otras ramas del conocimiento.

No ha existido una organización de estudiantes que convoque a una rebelión continental generalizada, pero los movimientos no son acontecimientos aislados. La toma de las universidades en Argentina en I9I8 tuvo un alto impacto para la búsqueda de la autonomía en todas las universidades de América Latina, vinculándose de manera entrecruzada con el movimiento por la autonomía de la UNAM (desde entonces: Universidad Nacional Autónoma de México, durante la presidencia de Emilio Portes Gil, en 1928, y logrando el reconocimiento oficial de dicha autonomía en 1929, lo cual, desde luego, se presentó como idea y propuesta de la misma presidencia).

A su vez, cada una de estas agitaciones estudiantiles llevó aparejados movimientos políticos - pugnas por el poder en el gobierno- y culturales -cualquier reforma en el organismo que representa la máxima autoridad en el conocimiento dentro del Estado, afecta la forma misma de entender, interpretar, validar y/o hacer cultura-.

\section{La Preparatoria de la UACH y el movimiento estudiantil}

Por la necesidad de realizar un abordaje interdisciplinario y de caso, llegamos al estudio del legado del pensamiento crítico y humanista de la Escuela Preparatoria de la Universidad de Chihuahua, ubicado dentro de los movimientos estudiantiles como un fenómeno que, a la 
vez de ser réplica, impactó también en diferentes ámbitos de estudio en México, así como los ya mencionados en América Latina.

El estudio de los conflictos estudiantiles nos debe poner a considerar si dicho conflicto es simplemente una reforma universitaria o en realidad se trata de una politización social que se expresa en el estudiantado. Si ocurre lo último, se manifiesta este intento de transformación social, entendiendo que los conflictos universitarios difícilmente se disocian de la vida pública de un país, por el contrario, están fuertemente unidos a las políticas nacionales. Es la complejidad del movimiento estudiantil y su estrecha relación con la lucha de clases lo que hace interesante su estudio.

En el núcleo de los movimientos estudiantiles se van forjando personajes políticos que tendrán peso en las decisiones del país, donde se desarrolla el conflicto "La educación universitaria garantizaría a sus hijos el ingreso a campos del saber y de influencia, especialmente en la política" (Marsiske, 20II, p. 9). En los movimientos estudiantiles se van constituyendo actores políticos que pueden llegar a formar parte del Estado o que, en su momento, tienen influencia en grupos de resistencia preexistentes. El Ejército Zapatista de Liberación Nacional (EZLN) podría ser un ejemplo claro de esto último. El conflicto universitario es también un campo de experimentación, un laboratorio social y plaza dentro de cada lucha de poder que implique los poderes del Estado.

Un problema fundamental dentro de las demandas universitarias,

52 advertido en estos estudios de la lucha estudiantil, es la tentativa de los estudiantes por realizar reformas dentro de las universidades; patente es también la reacción de rechazo a priori de las autoridades a las exigencias estudiantiles. En donde sea que se presente este fenómeno, los gobiernos por razones distintas, que siempre incumben a preservar las formas del poder de Estado legítimo que abanderan, prefieren negar 
estas reformas universitarias y proponen las propias, presentándolas como una alternativa legítima (o proponen las estudiantiles como propias -acabamos de citar un caso capital- de creerlo conveniente).

Esta lucha de intereses Universidad-Estado genera una radicalización en ambos campos: el Estado vira hacia el autoritarismo y el estudiantado, de entrada, integrado por reformistas demócratas, hacia una izquierda radical. "En definitiva, 'radicalización' remite a un proceso de acumulación política, de cuerpos e ideas condensados en colectivos humanos que las llevan a la práctica" (Califa, 20I4, párr. 9).

En el caso de la Preparatoria de la Universidad de Chihuahua, los estudiantes, junto a los universitarios, buscaban reformas a la Universidad y a la Preparatoria, que podemos cotejar con un artículo reciente del Dr. Víctor Orozco, donde manifiesta algunas exigencias del pliego petitorio de aquellos años:

"La Universidad debe Transformarse", el documento aludido al principio condensaba las banderas estudiantiles y académicas en general junto con las aspiraciones igualitarias o justicieras con las cuales se identificaban los movimientos estudiantiles de todo el orbe. Se demandaba educación superior para los marginados o impedidos de llegar a las instituciones, instrumentando para ellos amplios programas de becas e internados estudiantiles, reglas democráticas dentro de aquellas para designar a sus órganos de gobierno y diseñar programas de estudios, transparencia en el uso de los fondos, exigencia a los gobiernos para que suministraran recursos suficientes a la educación, entendida como una inversión y no como un gasto. Fue una lucha noble y audaz en sus aspiraciones. Por eso, atrajo la adhesión de miles de estudiantes y ciudadanos [Orozco, 20I7, párr. 9]. 
Los movimientos surgen de una necesidad, cuando las condiciones económicas comienzan a representar un problema para un determinado sector social. En torno a esta problemática, la organización va surgiendo como una solución previa, las discusiones al interior lo llevan entonces al nivel de la política; pero este nivel e interés comienza a tener un choque con la política oficial: es cuando se da el conflicto social o lucha de clases más intensa. En las agitaciones estudiantiles, la situación es la misma y es más fácil observarlo, debido en parte a que los movimientos estudiantiles comienzan con peticiones básicas, como la impartición de una cátedra o la elección democrática de directivos y docentes; es decir, la mayoría de los movimientos estudiantiles comienzan con peticiones localizadas que se confinan a una escuela, facultad o universidad, pero estos intereses chocan con los de las autoridades y -por ende- con los intereses del Estado. Esto que a primera vista parece intrascendente, subestimado, comienza a radicalizarse a tal punto que se convierte en bandera de lucha y entonces las pequeñas exigencias -que sólo se discutían en los pasillos, entre alumnos, maestros y administrativos- son llevados hasta las autoridades, empezando a fundirse cada vez más con reglamentos y leyes escolares, a tal punto que estas demandas, terminan conglomerando alumnos, organizándolos y llevándolos a convertirse en un movimiento.

Por su composición heterogénea y conflictos al interior, los movimientos estudiantiles son aislados y arrinconados, como parte de 54 una política que obedece a una necesidad de las autoridades para mantener su imagen de representar un gobierno fuerte. La lucha entre autoridad y estudiantes parece seguir siempre el mismo patrón. La dinámica automática va generando que la misma organización estudiantil comience a radicalizarse, a tal grado que los estudiantes que habían mantenido posiciones progresistas, moderadas, liberales y 
democráticas, al percibir la negativa de la autoridad, giran a posiciones de izquierda cada vez más radicales, allegando a sus luchas internas demandas de tipo social. Es entonces cuando el conflicto toma dimensiones de carácter local, estatal, nacional e incluso internacional, como lo fue el Mayo Francés.

Estos movimientos que muchas veces son de carácter progresista, nacionalistas, liberales, incluso de corte religioso o simplemente socialdemócratas y que no trascienden de discusiones ideológicas, al ser coartados y subestimados por las autoridades académicas y por el Estado; en su radicalización hacia la izquierda, sus propuestas pueden trascender hacia demandas de corte socialista y revolucionario. La transmutación política del movimiento es un problema que el mismo Estado parece estar dispuesto a asumir. Califa afirma que "la mayor parte de las indagaciones sobre el movimiento estudiantil en todo el mundo se produjeron en estos años, atraídas por tal proceso de radicalización que alcanzó niveles inéditos, nunca más superados” (Califa, 20I4, párr. 2).

Las contradicciones entre estudiantes y autoridades tienden a repetirse una y otra vez, a través del tiempo, en diferentes zonas geográficas, precisamente porque las autoridades difícilmente cederán ante las demandas estudiantiles por distintas condiciones. Como ya se mencionó, se debe mantener la figura de autoridad, que incluso tiene un status por encima de la del docente y los administrativos, en una estrecha relación con los puestos directivos y la rectoría que son puestos de carácter político y los que recae la responsabilidad de llevar a cabo los dictámenes gubernamentales y donde se verifica que se lleven a cabo los planes y programas nacionales o estatales, así como los reglamentos. Otra condición es que las propuestas hechas por los estudiantes, en su anhelo de reforma, no responden a los intereses de las autoridades; muchas veces, la transparencia, como prácticas 
efectivas de rendición de cuentas o las políticas democráticas, son asuntos que para las autoridades representan una debilidad en su capacidad de controlar el poder político sobre las instituciones educativas.

La rígida permanencia de una postura aparentemente inmutable de las autoridades educativas ante las demandas estudiantiles, parece ser una constante en la mayoría de las instituciones de los niveles medio superior y superior, por lo que los conflictos serán inevitables, lo que parece obedecer a una ley. Las exigencias del estudiantado y la negativa de las autoridades son solo dos de los elementos para que un movimiento estudiantil surja; existen otros más que habría que considerar, como la politización, la desigualdad social, las revoluciones, las guerras, el apogeo económico, los movimientos culturales y artísticos, los cambios de paradigmas ideológicos y muchos otros que pueden combinarse. La unión estos elementos es desigual; a veces la combinación de la política con la economía es suficiente; en otras la ideología y la política; el contraste económico de un sector social que ostenta el capital mientras que otro carece de lo esencial; entre otros. La combinación de distintos elementos son en realidad lo que hace que cada movimiento tenga sus propias peculiaridades y características, diferenciadas de otras manifestaciones colectivas.

El movimiento estudiantil chihuahuense se vinculó a los conflictos sociales del Estado, como el Comité de Defensa Popular (CDP), las organizaciones guerrilleras y los mítines por la defensa de los derechos 56 humanos; se inspiró en las tendencias de orden nacional como el movimiento estudiantil del 68, pero también en los internacionales, como la Revolución Cubana, el Mayo Francés o la revuelta argentina que culminó en el Cordobazo. 
El contexto social alimenta los movimientos, vinculándose a agitaciones de carácter local, nacional e internacional, coyunturas que se realizan de manera consciente e inconsciente, ya que obedece a un tipo de efecto dominó. El movimiento argentino que parecía no guardar ninguna relación con el movimiento estudiantil chihuahuense, podría tenerlo. En los acontecimientos de I9I8, en Córdoba, inicia la pugna por la democratización de las universidades, situación que revoluciona toda la superestructura de las universidades en América Latina, pues este hecho será el iniciador de los movimientos estudiantiles en esta región del continente americano.

En el libro Reforma y Revolución se realiza el análisis de la radicalización del movimiento estudiantil argentino que transitó de una posición reformista a una de izquierda radical:

Más concretamente, este trabajo se propone desarrollar un saber riguroso acerca del proceso de radicalización política estudiantil hacia la izquierda del arco político acaecido en la Argentina, tomando como caso de estudio lo sucedido en la Universidad de Buenos Aires (UBA), la casa de estudios más grande del país [Califa, 20I4, Párr. I].

Es así como los movimientos estudiantiles no se tratan únicamente como accidentes aislados, con pretensiones políticas de determinado grupo. El análisis que se intenta realizar se apoya en trabajos de especialistas, dado el carácter social de la investigación, la forma de abordar estos temas es tratando los movimientos como fenómenos, los cuales tienden a repetirse en todas las sociedades modernas donde existan universidades o instituciones similares que otorguen un nivel superior de estudios. Se debe tener en cuenta que las manifestaciones son parte sustancial del desarrollo y emancipación de las instituciones (guardando el equilibrio de esta contradicción aparente) y que estas son 
fenómenos sociales que no se pueden, por tanto, suprimir del panorama social.

\section{Algunas interpretaciones de las clases medias y los movimientos estudiantiles}

Es importante señalar que la postura teórica adoptada en la tesis de Juan Sebastián Califa, se encuentra muy relacionada con el paradigma marxista. El movimiento estudiantil es abordado desde una perspectiva de lucha de clases. En esta investigación se mencionan algunas contradicciones a la que llegaron estudiosos de este proceso político, como Jorge Graciarena y Juan Carlos Portantiero, quienes a partir de las clases medias intentaron explicar el fenómeno estudiantil. Las primeras manifestaciones que se realizaron entre los estudiantes de la UBA y de la Universidad de Córdoba tienen una relación directa con jóvenes que pertenecían casi en su totalidad a la clase media. Es por eso que sus primeras demandas fueron de tipo reformista, pero conforme se van desarrollando los procesos políticos en Argentina, estos estudiantes van teniendo posiciones cada vez más radicales, que los llevan a identificarse más con la clase proletaria que con la media.

Tal y como ocurre entre los estudiantes de la Preparatoria y de la Universidad de Chihuahua en I973, que identificados con la clase media en un principio, demandaban una transformación reformista, pero conforme se fueron desarrollando los procesos políticos en el contexto social, fueron radicalizándose e identificándose cada vez más con los sectores populares, campesinos y obreros. Es por ello que "las clases sociales no existen sino en la lucha de clases y los enfrentamientos sociales constituyen precisamente la expresión de esta última, 
manifestación que por lo tanto supone la estructura social de la sociedad y sus transformaciones" (Califa, 20I4, párr. 25).

Renate Marsiske (2007) afirma que las clases medias son determinantes en los movimientos estudiantiles; las caracteriza de cierto modo como clases progresistas y nacionalistas, los pronunciamientos prospectivos de algunos autores... en el redescubrimiento de las clases medias como fuerza social indispensable para el desarrollo" (Marsiske, 20II, p. 7). El autor señala que estas clases medias ven en la educación universitaria, el acceso de los hijos a una oportunidad de acercamiento al conocimiento, la ocasión para obtener un buen empleo, así como movilidad social y sobre todo el acceso a la política o -mejor dicho- a puestos públicos en el gobierno. Se preparan académicamente para la vida laboral, pero también para la toma en las decisiones políticas del país. Esto lleva a la conclusión de que si la política es una parte de la formación en las universidades, no es casual que si ocurre un conflicto universitario tome tendencias políticas (Aguilar, 2000).

Renate Marsiske (20II) confiere la dirección política de los movimientos estudiantiles a las clases medias, pero aún y cuando en su mayoría los estudiantes pertenezcan a ellas, se encuentran elementos de la clase trabajadora. Si estos movimientos viran hacia posiciones de clase, puede ser debido a la influencia de la lucha obrera y a que en el proceso político las organizaciones estudiantiles van buscando cada vez más un acercamiento al movimiento de los trabajadores para concretar su triunfo. En la tesis de Marsiske se rescatan elementos muy importantes como son la politización y el aumento de las clases medias, las cuales fueron desplazadas de sectores meramente académicos y profesionistas, a sectores productivos como el comercial y la industria. 


\section{Conclusiones}

Este trabajo que se desprende de una investigación doctoral, se consiguió una aproximación epistemológica a las causas y porqués del origen de los movimientos estudiantiles, a través de la indagación en varios documentos de especialistas en el tema. Siendo congruentes con la historiografía crítica y el método sintético, se incorporó la heurística, una búsqueda intensiva de documentos que han dado cuenta de distintas manifestaciones estudiantiles, acaecidas básicamente durante los años sesenta y setenta, así como fuentes documentales, libros, artículos de revistas, crónicas y memorias.

El trabajo hermenéutico, en la interpretación de todas las posturas ideológicas y políticas, fue una tarea de análisis de las posiciones moderadas, hasta posiciones de la izquierda radical, que al comparar los episodios, conseguimos hacer un balance, corroborando y discriminando documentos, que pudieran ser más certeros en sus conclusiones.

Conseguimos comprobar una de nuestras hipótesis, que aseguraba que los movimientos estudiantiles habían formado parte inherente de la formación universitaria. El caso de 1229 en Francia, del cual se tiene el primer registro de un enfrentamiento de estudiantes de la universidad de París con la policía, tuvo como consecuencia una huelga general.

Encontramos que los acontecimientos que siguieron a la Revolución Francesa, produjeron los primeros enfrentamientos de los estudiantes con las autoridades universitarias en el año de I829 y que, a partir de esta conmoción en París, comenzó una nueva etapa de los movimientos estudiantiles de la historia contemporánea. Esto coincide con lo que señalan algunos analistas que aseveran que las ideas libertarias fueron producto de la Ilustración, surgidas de la Revolución Francesa, mismas 
que se expandieron por toda Europa, Estados Unidos y América Latina, propagándose también en el continente asiático.

En cuanto al legado de la autonomía universitaria latinoamericana, fue básicamente la investigación de George R. Waggoner, la que nos llevó a concluir el porqué del compromiso social de estas universidades como resultado de la tradición de Salamanca y Alcalá. También percibimos que la diferencia sustancial en el sistema de gobierno universitario norteamericano dependen directamente del Estado y su tradición heredada de la universidad de París y Bolonia.

Una controversia que queda pendiente en esta investigación es que si bien es cierto que el movimiento de reforma universitaria cordobés de I9I8 no tiene una relación directa con los movimientos estudiantiles que se desarrollaron posteriormente en América Latina, tampoco es posible asegurar que se encuentren del todo desvinculados.

Creemos haber dado respuesta a la pregunta ¿Por qué se origina una manifestación estudiantil? Todo surge por peticiones muy moderadas, concernientes en un primer momento sólo a la Universidad, por ejemplo: la petición de un maestro para impartir una catedra, pero si estas peticiones son ignoradas o menospreciadas por las autoridades responsables, puede convertirse en un movimiento que aglutina a varios sectores populares. 


\section{Referencias Bibliográficas}

Aguilar Mora, M. (200o). El escándalo del Estado: una teoría del poder político en México. México, D.F.: Fontamara.

Califa, J. S. (20I4). Reforma y revolución: La radicalización política del movimiento estudiantil de la UBA 1943-I966. Buenos Aires: Eudeba.

González, D. S. (2010). ¿Cómo ha sido la vuelta?” Educación y Ciudad, I8, II3-I28.

IISUE. (24 de Noviembre de 2015). Los movimientos estudiantiles en América Latina [Archivo de video]. Recuperado de https://www.youtube.com/watch?v=JZU45L7LmAs

Marsiske, R. (20II). Universidades, clases medias y movimientos estudiantiles en América Latina (I9I8-I929). México: UNAM.

Orozco, V. (20I7). "La Universidad debe transformarse." El Diario.Mx. Recuperado de http://diario.mx/Opinion/20I7-05o6_oefb4593/la-universidad-debe-transformarse/

Silva, H., y Sonntag, R. H. (1970). Universidad, dependencia y revolución. (C. M. 33, Ed.) (Ist ed.). México, D.F.: Siglo XXI.

Waggoner, G. R. (1973). La Educación Superior en Estados Unidos y en Latinoamérica, I-I4. Recuperado de http://publicaciones.anuies.mx/revista/II/I/I/es/la-educacionsuperior-en-estados-unidos-y-en-latinoamerica

\footnotetext{
I "El origen del vocablo "bonapartismo" está en la experiencia de las dictaduras burguesas francesas del primer Napoleón, apellidado Bonaparte, y la de su "sobrino" Napoleón III. Marx y Engels denominaron con este título los regímenes despóticos, encarnados en un dictador, pero no necesariamente sólo en ellos, (también en
} 
camarillas, pequeñas oligarquías), en los que la burguesía acepta un régimen no democrático para garantizar el libre y amplio desarrollo del capitalismo" (Aguilar, 2000).

Este artículo se publica bajo una licencia de Creative Commons Reconocimiento-NoComercial 4.0 Internacional, y puede ser usados gratuitamente para fines no comerciales, dando los créditos a los autores y a la revista.

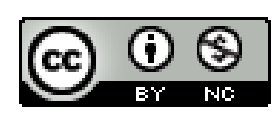


64 